\title{
Granzyme A as a potential biomarker of Mycobacterium tuberculosis infection and disease
}

\author{
Giuliana Guggino $^{\mathrm{a}, \mathrm{b}, 1}$, Valentina Orlando ${ }^{\mathrm{a}, \mathrm{b}, 1}$, Stella Cutrera ${ }^{\mathrm{a}, \mathrm{b}}$, Marco P. La Manna ${ }^{\mathrm{a}, \mathrm{b}}$, \\ Diana Di Liberto ${ }^{a, b}$, Valentina Vanini $^{c}$, Elisa Petruccioli ${ }^{c}$, Francesco Dieli $^{\mathrm{a}, \mathrm{b}}$, Delia Goletti ${ }^{\mathrm{c}}$, \\ Nadia Caccamo ${ }^{\mathrm{a}, \mathrm{b}, *}$ \\ a Central Laboratory of Advanced Diagnosis and Biomedical Research (CLADIBIOR), Università di Palermo, Palermo, Italy \\ ${ }^{\mathrm{b}}$ Dipartimento di Biopatologia e Biotecnologie Mediche, Università di Palermo, Palermo, Italy \\ ${ }^{\mathrm{c}}$ Institute for Infectious Diseases L. Spallanzani, Rome, Italy
}

\section{A R T I C L E I N F O}

\section{Article history:}

Received 1 April 2015

Received in revised form 12 May 2015

Accepted 27 May 2015

Available online 5 June 2015

\section{Keywords:}

Granzyme A

IFN- $\gamma$

IGRA tests

Tuberculosis

Infection

Disease

\begin{abstract}
A B S T R A C T
Cytotoxic molecules such as granulysin, perforin and granzymes produced by cytolytic $\mathrm{T}$ cells directly contribute to immune defense against tuberculosis (TB). In search for novel TB biomarkers, we have evaluated the levels of granzyme A in plasma obtained from QuantiFERON-TB Gold In tube (QFT-IT) assays from patients with active TB disease and subjects with latent TB infection (LTBI).

Granzyme A serum levels in TB patients were significantly lower than values found in LTBI subjects even after subtraction of the unstimulated levels from the antigen-stimulated responses. The receiver operator characteristics (ROC) curve analysis comparing TB patients and LTBI groups, showed that at a cut-off value of granzyme A of $<3.425 \mathrm{pg} / \mathrm{ml}$, the sensitivity and the specificity of the assay were $29.41 \%$ and $94.74 \%$, respectively.

Our results suggest that granzyme A could be considered another biomarker of TB, that can be used, other than IFN- $\gamma$, to discriminate between patients with active TB and LTBI subjects in a well characterized cohort of confirmed Mycobacterium tuberculosis-infected individuals.
\end{abstract}

(c) 2015 European Federation of Immunological Societies. Published by Elsevier B.V. All rights reserved.

\section{Introduction}

With 1.4 million deaths and 9 million cases per year, tuberculosis (TB) remains a global health care problem and control of the global TB epidemic has been impaired by the lack of an effective vaccine, by the emergence of drug resistant forms of Mycobacterium tuberculosis (Mtb) and by the lack of sensitive and rapid diagnostics [1-3]. It is estimated, by epidemiological reports, that one-third of the world's population is latently infected with Mtb, but the majority of infected individuals develop long-lived protective immunity, which controls and contains Mtb in a T cell-dependent manner. Development of TB disease results from interactions among the environment, the host, and the pathogen, and known risk factors include HIV co-infection, immunodeficiency, diabetes mellitus, overcrowding, malnutrition, and general poverty; therefore, an

\footnotetext{
* Corresponding author at: Department of Biopathology, Corso Tukory 211, 90134 Palermo, Italy. Tel.: +39 091 6555940; fax: +39 0916555901.

E-mail address: nadia.caccamo@unipa.it (N. Caccamo)

1 These authors contributed equally to this work.
}

effective T cell response determines whether the infection resolves or develops into clinically evident disease.

The diagnosis of TB disease remains a challenge in resourceconstrained settings [1]. In fact, although the analysis of the sputum is widely used for the diagnosis of active TB, Mtb is not always detectable in the sputum as occurs in, such individuals with extra-pulmonary TB (EPTB) and children $[2,4]$, making this test low sensitive. Therefore, EPTB and smear-negative pulmonary TB (PTB) are usually diagnosed based on clinical symptoms. The gold standard remains the isolation and culture of the bacilli from sputum but this requires long time [5]. Interferon gamma (IFN- $\gamma$ ) release assays (IGRAs) have added a new tool for the immune-based diagnosis of TB, as these tests are highly specific and accurate in the diagnosis of Mtb infection, especially when compared to the skin test [6]. The limits of these tests are represented by the inability to discriminate between subjects with active TB disease and subjects with latent infection [1,6,7]. Therefore, several Mtb specific antigens [8,9], and host markers other than IFN- $\gamma$ in Mtb-specific antigen-stimulated whole blood culture assays have been investigated for TB diagnostic purposes [10,11]. The need for biomarkers extends beyond the urgency for improved diagnostic tools: the assessment of the disease status and of the risk of progression to 
Table 1

Characteristics of study groups.

\begin{tabular}{|c|c|c|c|c|c|}
\hline \multirow[t]{2}{*}{ Enrolled subjects (\%) } & Healthy donors & PnI & LTBI & Active TB & Total \\
\hline & $\begin{array}{l}N=18 \\
(22.2)\end{array}$ & $\begin{array}{l}N=10 \\
(12.3)\end{array}$ & $\begin{array}{l}N=19 \\
(23.4)\end{array}$ & $\begin{array}{l}N=34 \\
(42)\end{array}$ & $\begin{array}{l}81 \\
(100.0)\end{array}$ \\
\hline $\begin{array}{l}\text { Median age } \\
\text { (IQR) } \\
\text { Male gender }(\%)\end{array}$ & $\begin{array}{l}32.5 \\
(21-59) \\
8(44.4)\end{array}$ & $\begin{array}{l}41 \\
(30-56) \\
6(60)\end{array}$ & $\begin{array}{l}32 \\
(16-61) \\
9(47.4)\end{array}$ & $\begin{array}{l}31 \\
(20-51) \\
26(76.5)\end{array}$ & $\begin{array}{l}32 \\
(16-61) \\
43(60.6)\end{array}$ \\
\hline $\begin{array}{l}\text { Origin } N(\%) \\
\text { Western Europe } \\
\text { Eastern Europe } \\
\text { Asia } \\
\text { Africa }\end{array}$ & $\begin{array}{l}18(100.0) \\
- \\
- \\
-\end{array}$ & $10(100.0)$ & $\begin{array}{l}12(63.2) \\
5(26.3) \\
- \\
2(10.5)\end{array}$ & $\begin{array}{l}6(17.6) \\
20(58.8) \\
5(14.7) \\
3(8.8)\end{array}$ & $\begin{array}{l}36(50.7) \\
25(35.2) \\
5(7.0) \\
5(7.0)\end{array}$ \\
\hline $\begin{array}{l}\text { BCG } N(\%) \\
\text { Vaccinated } \\
\text { Unvaccinated }\end{array}$ & $18(100.0)$ & $10(100.0)$ & $\begin{array}{l}7(36.8) \\
12(63.2)\end{array}$ & $\begin{array}{l}29(85.3) \\
5(14.7)\end{array}$ & $\begin{array}{l}38(53.5) \\
32(45.1)\end{array}$ \\
\hline $\begin{array}{l}\text { TST } N(\%) \\
\text { Positive } \\
\text { Negative } \\
\text { Unknown }\end{array}$ & $\begin{array}{l}- \\
18(100.0) \\
-\end{array}$ & $10(100.0)$ & $\begin{array}{l}7(36.8) \\
2(10.5) \\
10(52.6)\end{array}$ & $\begin{array}{l}12(35.3) \\
5(14.7) \\
17(50.0)\end{array}$ & $\begin{array}{l}19(26.8) \\
25(35.2) \\
27(38.0)\end{array}$ \\
\hline $\begin{array}{l}\text { Pulmonary TB diagnosis } \\
\text { Microbiological diagnosis } \\
\text { Pulmonary pneumococcal infection } \\
\text { Microbiological diagnosis }\end{array}$ & - & $10(100.0)$ & - & $\begin{array}{l}34(100.0) \\
-\end{array}$ & $\begin{array}{l}34(100.0) \\
-\end{array}$ \\
\hline
\end{tabular}

active disease or the assessment of treatment success early during therapy are critical bottlenecks in the development of new vaccines and drugs, and could allow categorization of therapy based on individual risk of unfavorable outcome. While, the necessity to discriminate between LTBI and active TB, clear-cut biological markers separating the two conditions are not yet available [12-14]. Biomarkers and surrogate endpoints are therefore crucial tools for the development of innovative strategies for TB management [15]. Since several reports on human and mouse TB have provided that several T cells use granule-dependent killing of Mtb-infected cells, in our study, we aimed to investigate whether granzyme A could be detected at the different phases of Mtb infection (LTBI, active TB) patients in order to use this molecule as specific signature for TB diagnosis.

\section{Materials and methods}

\subsection{Characteristics of the enrolled individuals}

81 individuals were prospectively enrolled as here reported: (a) healthy donors (HD): 18 individuals $\mathrm{PPD}^{-}$(8 men, 10 women, age range 21-59 years); (b) individuals with pulmonary pneumococcal infection (PnI): 10 individuals (6 men, 4 women, age range 30-56 years); (c) latent infection subsets (LTBI): 19 individuals (9 men, 10 women, age range 16-61 years) who reported household or equivalent close contact (work) with smear-positive pulmonary TB patients in the previous 3 months, tested TST and QFT-IT-positive, with negative chest radiographic results for pulmonary lesions and no prior preventive therapy; (d) active TB group: 34 individuals diagnosed with TB (either with a positive culture for Mtb from sputa or with positive Mtb-specific RNA amplification results from biopsy specimens and/or biological fluids) 26 men, 8 women (age range $20-51$ years) who started specific treatment $<8$ days before enrolment (see Table 1).

All the patients were recruited from the L. Spallanzani National Institute for Infectious diseases, Rome. TB-infected patients had clinical and radiological findings consistent with active pulmonary TB [16]. Diagnosis was confirmed by bacteriological isolation of Mtb in 25 patients. Patients who resulted negative to Mtb culture in sputa were classified as having "clinical TB" when (i) clinical, pathological and radiological findings consistent with TB were documented; (ii) an alternative diagnosis was excluded, and (iii) a full course of anti-TB treatment was started and an appropriate clinical/radiological response was obtained. All patients were treated in accordance with Italian guidelines and received therapy for 6 months. Treatment was successful in all participants, all of whom completed the full course of anti-TB chemotherapy, as evidenced by the absence of any clinical or radiographic evidence of recurrent disease and sterile mycobacterial cultures. Plasma obtained from cells stimulated for QFT-IT was collected. None of the TB patients had been vaccinated with BCG, or had evidence of HIV infection, or was being treated with steroid or other immunosuppressive or anti-tubercular drugs at the time of their first sampling. Tuberculin (PPD) skin tests were considered positive when the induration diameter was larger than $10 \mathrm{~mm}$ at $72 \mathrm{~h}$ since injection of 5 U of PPD (Statens Serum Institut, Copenhagen, Denmark). The study was approved by the Ethical Committee of the L. Spallanzani National Institute for Infectious diseases (INMI), approval number 2/2007, Italy, where the patients were recruited. Informed consent was written by all participants. Individuals with LTBI were defined as healthy people with a positive tuberculin skin test and QFT-IT test and no symptoms and signs of active TB. Among the HIVuninfected subjects the healthy donors were selected as subjects without reported exposure to Mtb and QFT-IT negative. Individuals who tested positive to HIV antibody (three subjects) or were on immunosuppressive drugs (two subjects) were not included in the study. Upon enrolment, demographic and epidemiological information was collected through a structured questionnaire. QFT-IT was performed using TB antigen (overlapping peptides from CFP-10 and ESAT-6 and TB7.7), mitogen- and unstimulated tube (nil).

\subsection{QuantiFERON TB-gold in tube (QFT-IT)}

QFT-IT (Cellestis Limited, Carnegie, Victoria, Australia) was performed using TB antigen-, mitogen- and unstimulated tube (nil) The tube containing TB antigen uses overlapping peptides from CFP-10 and ESAT-6 and TB7.7. The assay was performed and the results were scored as indicated by the manufacturer's instructions (the cut-off value for a positive test was $0.35 \mathrm{IU} / \mathrm{ml}$ ). An indeterminate score was assigned if the IFN- $\gamma$ response to the mitogen after subtracting the nil IFN- $\gamma$ response was $<0.5 \mathrm{IU} / \mathrm{ml}$ or if the nil IFN$\gamma$ response was $>8 \mathrm{IU} / \mathrm{ml}$. The reported results from QFT-IT were 

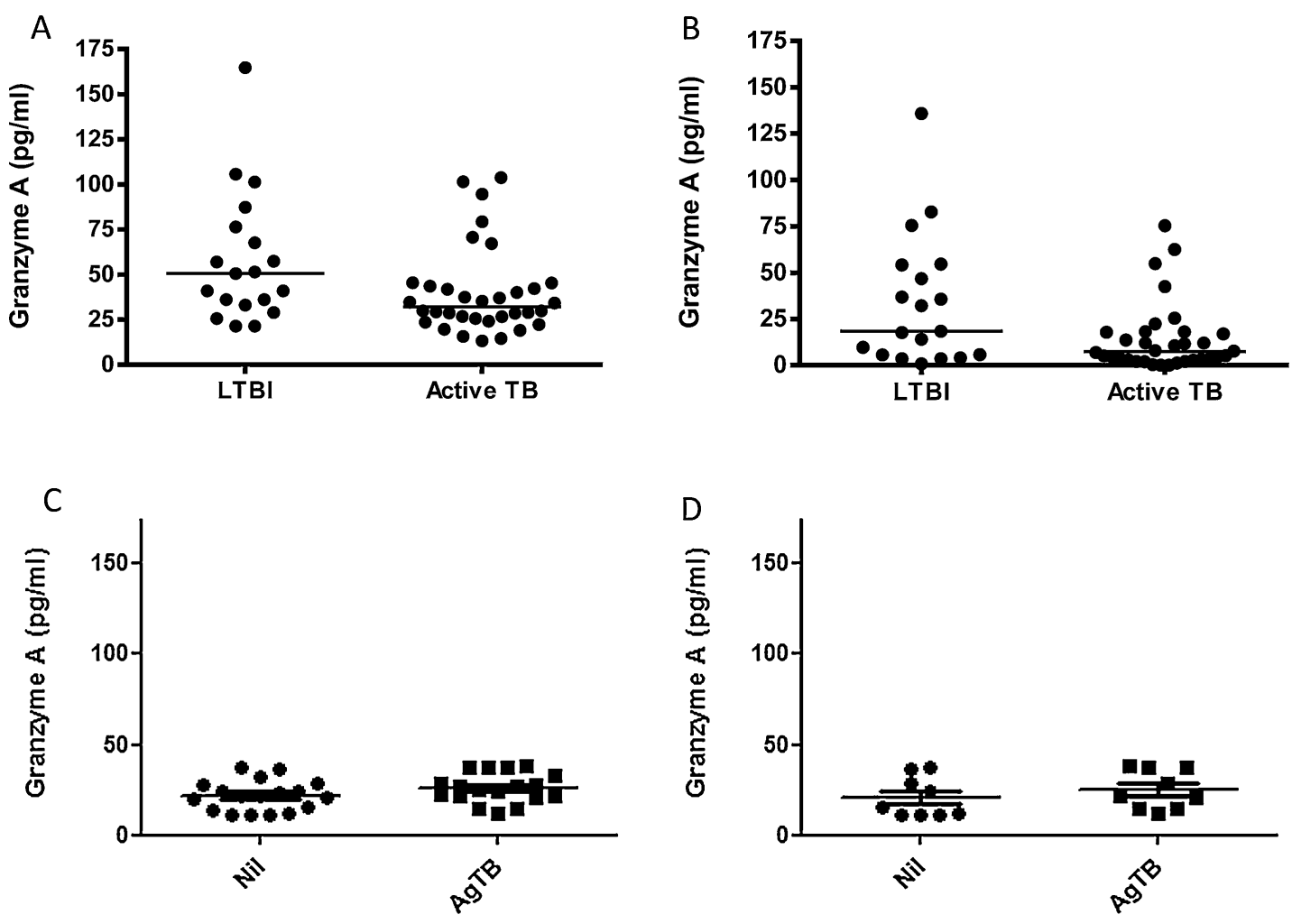

Fig. 1. Significance of granzyme A levels in plasma after whole blood stimulation in patients with active TB, LTBI subjects, healthy PPD ${ }^{-}$donors (HD).

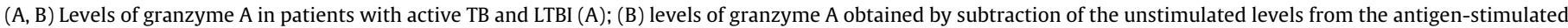

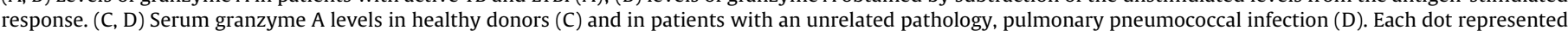
one individual. The horizontal bar indicated the median of each group. $p<0.05$ were considered statistically significant.

tabulated after the subtraction of the relative unstimulated control. The patients were analyzed for the IFN- $\gamma$ release using QFT-IT.

\subsection{Granzyme A-response based assay}

The level of granzyme A was measured in the plasma of QFTIT tubes using Human granzyme A ELISA assay (Bioscience, UK) according to the manufacturer's instructions. Plasma was diluted $1: 2$ and further dilutions were performed if necessary.

\subsection{Statistical analysis}

The mean values were calculated. Differences between two independent subjects groups were evaluated by Mann-Whitney test. Analysis was carried out with SPSS v.19 for Windows (SPSS Italia SRL, Bologna, Italy). All analyses were two sided, where a $p$ value $<0.05$ was considered as statistical significant. The accuracy of granzyme $A$ for the diagnosis of TB disease was estimated by performing receiver operator characteristics (ROC) curve analysis. Optimal cut-off values were selected based on the Youden's index, defined as the largest difference between the sensitivity and specificity, taken over all points on the ROC curve [17]. Data were analyzed using the Statistica software (Statsoft, Ohio, USA) and GraphPad prism, version 5.0 (GraphPad Software, San Diego, CA, USA).

\section{Results}

Serum granzyme A levels were compared in patients with active TB and LTBI subjects in order to find out differences that may improve the detection of the response to QFT-IT for immunodiagnosis of TB. Basal levels of granzyme A (i.e. in unstimulated plasma) were low and comparable in all tested groups.
Appreciable granzyme A levels were detected in TB antigensstimulated plasma: median granzyme A levels in TB patients were $31.97 \mathrm{pg} / \mathrm{ml}$ and this value was significantly different than median values found in LTBI subjects $(50.51 \mathrm{pg} / \mathrm{ml}, p<0.04)$ (Fig. 1A).

The antigen specific responses of granzyme A obtained by subtraction of the unstimulated levels from the antigen-stimulated response were analyzed in order to evaluate the contribution of unstimulated marker level to the diagnosis of TB infection/disease. As shown in Fig. 1B, differences between granzyme A median levels in LTBI subjects and patients with active TB were maintained and found still significant as previously reported $(p=0.02)$, even after subtraction of background levels.

Serum granzyme A levels were also analyzed in healthy donors and in patients with an unrelated pathology, pulmonary pneumococcal infection. Levels of granzyme A both in unstimulated and in Ag-TB-stimulated plasma were low in both tested groups, (Fig. 1C and D), thus indicating the specificity of the assay.

Of interest, results of antigen-specific levels of granzyme A were paralleled by data of antigen-specific production of IFN- $\gamma$ assessed by QFT (Fig. 2), which were also significantly lower in patients with active TB when compared to LTBI subjects (Fig. 2). However, as shown in Fig. 3, we did not find any significant correlation between specific levels of granzyme A and IFN- $\gamma$.

We assessed the diagnostic accuracies of granzyme A marker detected in overnight whole blood stimulation by ROC curve analysis. As shown in Fig. 4, the area under the ROC curve obtained comparing patients with active TB versus LTBI subjects was 0.6935 (95\% CI 0.5424-0.8446) with $p=0.02$. When a cut-off value of granzyme A of $<3.425 \mathrm{pg} / \mathrm{ml}$ was chosen for scoring purposes to maximize the sum of sensitivity and specificity, we found that the sensitivity and the specificity were $29.41 \%$ and $94.74 \%$ respectively, comparing active TB patients with LTBI subjects. These results 


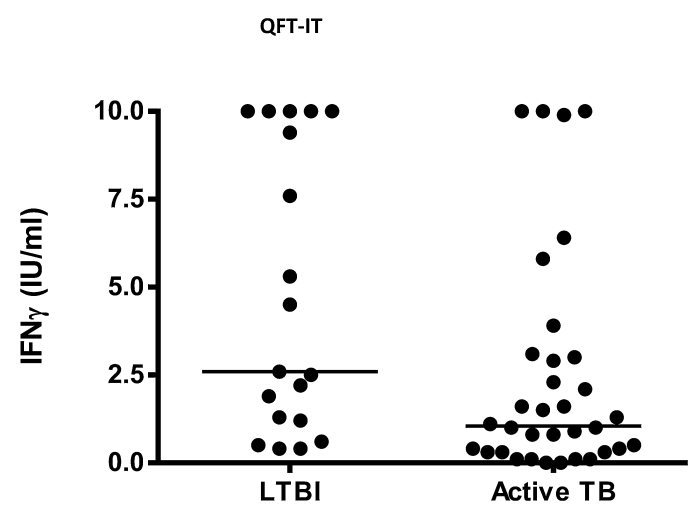

Fig. 2. IFN- $\gamma$ levels in plasma after whole blood stimulation in patients with active TB and LTBI subjects.

Each dot represented one individual. The horizontal bar indicated the median of each group. $p<0.05$ were considered statistically significant.

suggest that granzyme $\mathrm{A}$ could be considered an additional biomarker of TB infection/disease.

\section{Discussion}

In our study we have investigated the potential of granzyme A levels in plasma obtained from stimulated whole blood, as biomarker of infection/disease comparing patients with active TB disease with LTBI subjects.

IGRAs are well established and remain the tests of choice for the diagnosis of TB infection/disease in many settings. However, these assays do not discriminate between LTBI and active TB disease and are therefore not recommended in high burden settings [18]. Therefore, new diagnostic tests based on Mtb antigens and host markers would be mostly beneficial to TB control programs if such antigens and markers are incorporated into rapid, point-of-care test platforms.

Over the past decade both human and Mtb biomarker studies have focused on three specific areas of research: biomarkers predicting treatment efficacy and cure of active TB [19,20], the reactivation of latent tuberculosis infection, and the induction of protective immune responses by vaccination and drug trial design [21-23].

The current challenge is to identify markers that are specific and unique for TB that could represent a specific signature when compared with other infectious diseases which have high clinical similarity to TB.

Several groups of research still focus on assessing the quality of single biomarkers, there is an emerging interest in panels of biomarkers composed of multiple candidate targets which are

LTBI

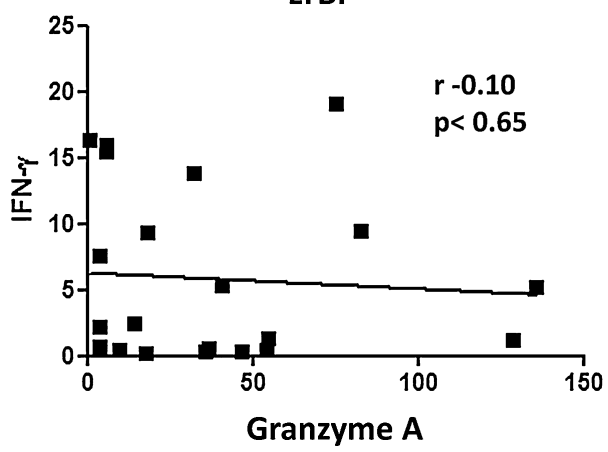

TB vs LTBI: ROC curve

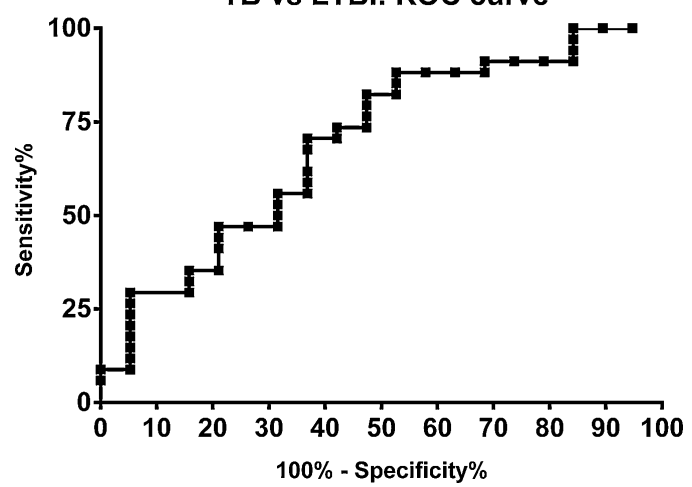

Fig. 4. Receiver operating characteristic (ROC) curve for the absolute value of granzyme A after antigen-specific stimulation. The solid line shows the result for the absolute values of granzyme A. Absolute values were calculated by subtracting the OD of the tube with mitogens and the negative control from the absorbance of the tube containing Mtb-antigens. The area under the curve (AUC) was 0.6935 (95\% CI 0.5424-0.8446) with $p<0.02$.

neither specific nor sensitive when used as single tests, but which show a good performance when used in combination. Different strategies have been employed in terms of improvements of the existing IGRA test, trying to incorporate additional Mtb antigens in order to improve diagnostic sensitivity of the tests without compromising specificity [24]. Moreover, measurement of different soluble molecules such as cytokines or chemokines or cytotoxic molecules in addition to IFN- $\gamma$ has also been proposed as a way of improving the diagnostic sensitivity of IGRAs [25]. Very recently, a review of 22 studies investigated on the role of IP-10 in TB diagnosis empathizing that its levels may be used to better detect TB infection in young children and in people with TB-HIV coinfection, where usually the counts of CD4T cells are very low [26].

Several groups have studied the role of different cytotoxic molecules, like granulysin, perforin and granzymes in TB. Worku and Hoft have previously demonstrated that increased mRNA expression of perforin, granzyme and granulysin contribute to a protective host response in human TB [27]. This improved mechanism of protection has also been demonstrated using antibody blockage of the granule exocytosis pathway, that dramatically reduced the lytic activity of $T$ cells against Mtb-infected macrophages and the increase of intracellular growth of bacteria [28]. Similarly, granulysin expression by $\mathrm{CD} 4^{+}$memory $\mathrm{T}$ cells is a candidate immune marker for TB infection, notably, in childhood and adolescence [29] and CD4T cells expressing granulysin correlate with the clinical forms of leprosy [30]. Our own previous studies demonstrated significantly lower plasma granulysin levels in adults with active disease in highly endemic area in Indonesia,

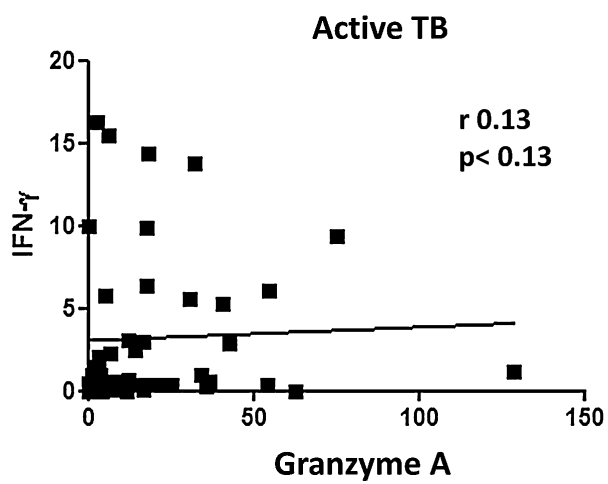

Fig. 3. Correlation between levels of IFN- $\gamma$ and granzyme A in patients with active TB and LTBI subjects.

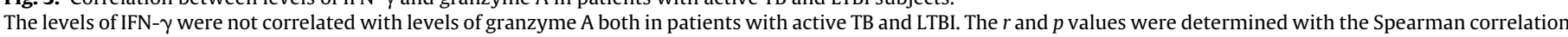
coefficient. 
and in children with TB disease in Italy [31], which increased after completion of therapy. An important aspect of that study was that the granulysin levels did not correlate with IFN- $\gamma$ levels suggesting that their cellular source in part was not overlapping [32].

Recently, Pitabut et al. have evaluated granulysin, perforin, granzyme-B and IFN- $\gamma$ levels by ELISA in patients with pulmonary TB with and without HIV coinfection. They found that circulating granulysin and perforin levels in TB patients were lower than healthy controls, whereas the granulysin and perforin levels in TBHIV coinfected patients were much higher than in any other groups, suggesting activation of the immune system in TB-associated HIV infection [33,34].

Results here reported show that serum granzyme A levels were significantly lower in patients with active TB disease compared to LTBI subjects. Based on these data, we performed a ROC analysis using TB patients and LTBI (Fig. 4). Significant results for area under the curve (AUC) analysis were obtained. For scoring purposes we chose a cut-off to maximize the sum of sensitivity and specificity. We found that a granzyme A cut-off $<3.425 \mathrm{pg} / \mathrm{ml}$ predicted a discrimination between active TB patients and LTBI subjects with $29.4 \%$ sensitivity and $94.7 \%$ specificity. The value of ROC curve highlighted that the test has poor sensitivity but is highly specific, which means that there is high probability that a patient with active TB is negative to the test and that LTBI subject is found positive to the test. On the other hand, the low level of sensitivity, could represent a limit of the test. Exploiting the very high specificity of the granzyme A assay, clearly points to this molecule as a potential biomarker of TB infection/disease; moreover, we would like to stress the point that this is a pilot study which presents limitations that will require additional work to be overcome. Above all, the study was performed on a relatively small number of individuals and certainly the research would benefit from a larger study cohorts.

In conclusion, our results suggest that granzyme A could be considered a new additional biomarker of TB, that can be used, other than IFN- $\gamma$, to identify subjects infected by Mtb with the limit due to not discriminate between active TB and LTBI.

Further studies are thus required to confirm these data in a larger number of patients and to evaluate the possible implications in terms of TB risk.

\section{Acknowledgments}

This work was supported by grants from the Norwegian Agency for Development Cooperation (NORAD) through Tuberculosis Vaccine Initiative (TBVI) contract no. 1001143436007833, the European Commission within the 7th Framework Programme, NEWTBVAC contract no. HEALTH-F3-2009-241745 and the Horizon2020 Programme, TBVAC2020 contract no. 643381. The text represents the authors' views and does not necessarily represent a position of the European Commission, which will not be liable for the use made of such information.

\section{References}

[1] World Health Organization, Global Tuberculosis Report 2012: WHO Fact Sheet Number 104, 2012, Available: http://www.who.int/tb/publications/ factsheet_global.pdf

[2] N.N. Chegou, K.G. Hoek, M. Kriel, R.M. Warren, T.C. Victor, G. Walzl, Tuberculosis assays: past, present and future, Exp. Rev. Anti Infect. Ther. 4 (2011) 457-469.

[3] WHO, Global Tuberculosis Report 2011, WHO Press, Geneva, 2011, Available from: http://whqlibdoc.who.int/publications/2011/9789241564380_eng.pdf.

[4] H.S. Whitworth, M. Scott, D.W. Connell, B. Dongés, A. Lalvani, IGRAs - the gateway to T cell based TB diagnosis, Methods 61 (2013) 52-62.

[5] K.E. Verweij, A.R. Kamerik, J. van Ingen, J.H. van Dijk, P. Sikwangala, P. Thuma, et al., Application of modern microbiological diagnostic methods for tuberculosis in Macha, Zambia, Int. J. Tuberc. Lung Dis. 14 (2010) 1127-1131.
[6] D.W. Dowdy, M.C. Lourenço, S.C. Cavalcante, V. Saraceni, B. King, J.E. Golub, et al., Impact and cost-effectiveness of culture for diagnosis of tuberculosis in HIV-infected Brazilian adults, PLoS ONE 3 (2008) e4057.

[7] S. Sester, G. Sotgiu, C. Lange, C. Giehl, E. Girardi, G.B. Migliori, et al., Interferon- $\gamma$ release assays for the diagnosis of active tuberculosis: a systematic review and meta-analysis, Eur. Respir. J. 37 (2011) 100-111.

[8] M. Pai, D. Menzies, The new IGRA and the old TST: making good use of disagreement, Am. J. Respir. Crit. Care Med. 175 (2007) 529-531.

[9] N.N. Chegou, G.F. Black, A.G. Loxton, K. Stanley, P.N. Essone, M.R. Klein, et al., Potential of novel Mycobacterium tuberculosis infection phase-dependent antigens in the diagnosis of TB disease in a high burden setting, BMC Infect. Dis. 12 (2012) 10

[10] D.P. Dosanjh, M. Bakir, K.A. Millington, A. Soysal, Y. Aslan, S. Efee, et al., Novel M tuberculosis antigen-specific T-cells are early markers of infection and disease progression, PLoS ONE 6 (2011) e28754.

[11] N.N. Chegou, G.F. Black, M. Kidd, P.D. van Helden, G. Walzl, Host markers in QuantiFERON supernatants differentiate active TB from latent TB infection: preliminary report, BMC Pulm. Med. 9 (2009) 21.

[12] N.N. Chegou, P.N. Essone, A.G. Loxton, K. Stanley, G.F. Black, G.D. van der Spuy, et al., Potential of host markers produced by infection phase-dependent antigen-stimulated cells for the diagnosis of tuberculosis in a highly endemic area, PLoS ONE 7 (2012) e38501.

[13] U. Mack, G.B. Migliori, M. Sester, H.L. Rieder, S. Ehlers, D. Goletti, et al., LTBI: latent tuberculosis infection or lasting immune responses to M tuberculosis? A TBNET consensus statement, Eur. Respir. J. 33 (2009) 956-973.

[14] G. Walzl, K. Ronacher, W. Hanekom, T.J. Scriba, A. Zumla, Immunological biomarkers of tuberculosis, Nat. Rev. Immunol. 11 (2011) 343-354.

[15] L. Norbis, P. Miotto, R. Alagna, D.M. Cirillo, Tuberculosis: lights and shadows in the current diagnostic landscape, New Microbiol. 36 (2013) 111-120.

[16] R.S. Wallis, M. Pai, D. Menzies, T.M. Doherty, G. Walzl, M.D. Perkins, et al., Biomarkers and diagnostics for tuberculosis: progress, needs, and translation into practice, Lancet 375 (2010) 1920-1937.

[17] Anon., Diagnostic standards and classification of tuberculosis in adults and children: this official statement of the American Thoracic Society and the Centers for Disease Control and Prevention was adopted by the ATS Board of Directors, July (1999), Am. J. Respir. Crit. Care Med. 161 (2000) 1376-1395

[18] R. Fluss, D. Faraggi, B. Reiser, Estimation of the Youden Index and its associated cutoff point, Biomed. J. 47 (2005) 458-472.

[19] World Health Organization, Use of Tuberculosis Interferon - Gamma Release Assays (IGRAs) in Low - and Middle - Income Countries: Policy Statement, 2011.

[20] J. Maertzdorf, J. Weiner 3rd, S.H. Kaufmann, Enabling biomarkers for tuberculosis control, Int. J. Tuberc. Lung Dis. 16 (2012) 1140-1148.

[21] J. Weiner, J. Maertzdorf, S.H. Kaufmann, The dual role of biomarkers for understanding basic principles and devising novel intervention strategies in tuberculosis, Ann. N. Y. Acad. Sci. 1283 (2013) 22-29.

[22] B. Pulendran, S. Li, H.I. Nakaya, Systems vaccinology, Immunity 33 (2010) 516-529.

[23] A. Diercks, A. Aderem, Systems approaches to dissecting immunity, Curr. Top. Microbiol. Immunol. 363 (2013) 1-19.

[24] M. Mooney, S. McWeeney, R.P. Sekaly, Systems immunogenetics of vaccines, Semin. Immunol. 25 (2013) 124-129.

[25] D.P. Dosanjh, T.S. Hinks, J.A. Innes, J. Deeks, G. Pasvol, et al, Improved diagnostic evaluation of suspected tuberculosis, Ann. Intern. Med. 148(2008) 325-336

[26] M. Ruhwald, M.G. Aabye, P. Ravn, IP-10 release assays in the diagnosis of tuberculosis infection: current status and future directions, Expert Rev. Mol. Diagn. 12 (2012) 175-187.

[27] S. Worku, D.F. Hoft, Differential effects of control and antigen-specific T cells on intracellular mycobactrerial growth, Infect. Immun. 71 (2003) 1763-1773.

[28] D.H. Canaday, R.J. Wilkinson, O. Li, C.V. Harding, R.F. Silver, W.H. Boom, CD4(+) and CD8(+)T cells kill intracellular Mycobacterium tuberculosis by a perforin and Fas/Fas ligand-independent mechanism, J. Immunol. 167 (2001) 2734-2742.

[29] H. Mueller, K.C. Faé, K. Magdorf, C.A. Ganoza, U. Wahn, U. Guhlich, et al., Granulysin-expressing CD4+ T cells as candidate immune marker for tuberculosis during childhood and adolescence, PLoS One 12 (2011) e29367.

[30] M.T. Ochoa, S. Stenger, P.A. Sieling, S. Thoma-Uszynski, S. Sabet, S. Cho, et al., T-cell release of granulysin contributes to host defense in leprosy, Nat. Med. 2 (2001) 174-179.

[31] E. Sahiratmadja, B. Alisjahbana, S. Buccheri, D. Di Liberto, T. de Boer, I. Adnan, et al., Plasma granulysin levels and cellular interferon-gamma production correlate with curative host responses in tuberculosis, while plasma interferon-gamma levels correlate with tuberculosis disease activity in adults, Tuberculosis (Edinb.) 87 (2007) 312-321

[32] D. Di Liberto, S. Buccheri, N. Caccamo, S. Meraviglia, A. Romano, P. Di Carlo, et al. Decreased serum granulysin levels in childhood tuberculosis which reverse after therapy, Tuberculosis (Edinb.) 4 (2007) 322-328.

[33] N. Pitabut, S. Sakurada, T. Tanaka, C. Ridruechai, J. Tanuma, T. Aoki, et al., Potential function of granulysin, other related effector molecules and lymphocyte subsets in patients with TB and HIV/TB coinfection, Int. J. Med. Sci. 10 (2013) 1003-1014.

[34] N. Pitabut, S. Mahasirimongkol, H. Yanai, C. Ridruechai, S. Sakurada, P. Dhepakson, et al., Decreased plasma granulysin and increased interferon-gamma concentrations in patients with newly diagnosed and relapsed tuberculosis, Microbiol. Immunol. 55 (2015) 565-573. 